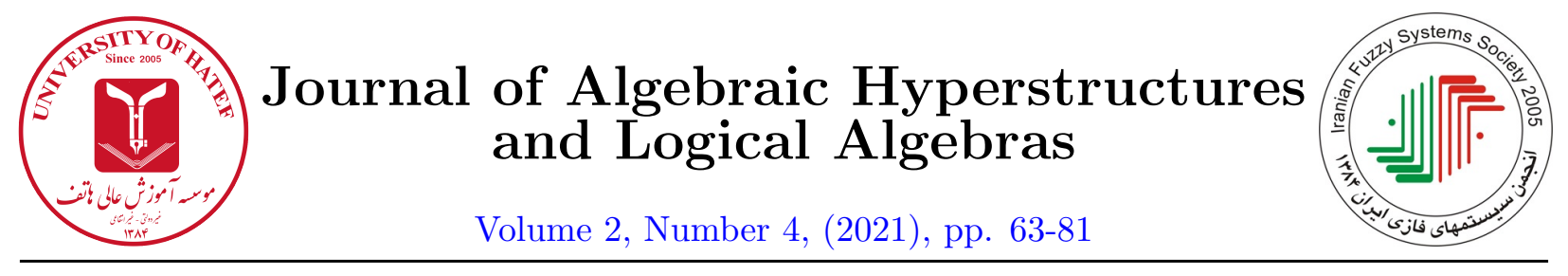

\title{
Neutrosophic soft metric matrices with applications in decision-making
}

\author{
M. Khan ${ }^{1}$, M. Zeeshan ${ }^{2}$ and S. Iqbal ${ }^{3}$ \\ ${ }^{1}$ Department of Mathematics, COMSATS University Islamabad, Abbottabad Campus, Pakistan \\ ${ }^{2,3}$ Department of Mathematics, COMSATS University Islamabad, Islamabad Campus, Pakistan \\ madadmath@yahoo.com, zeeshan.msc08@gmail.com, soh.iqbal@gmail.com
}

\begin{abstract}
In this paper, we introduce neutrosophic soft metric matrices and define some new operations on these matrices. Moreover, we develop an algorithm using neutrosophic soft metric matrices and apply it to a decision-making problem.
\end{abstract}

\section{Article Information}

Corresponding Author:

M. Zeeshan;

Received: May 2021;

Revised: June 2021;

Accepted: July 2021;

Paper type: Original.

\section{Keywords:}

Soft set, neutrosophic set, neutrosophic soft set, neutrosophic soft metric matrices.

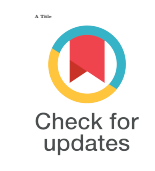

\section{Introduction}

Fuzzy sets (FS) [32], complex fuzzy sets (CFS) [28], intuitionistic fuzzy sets (IFSs), soft sets [4], fuzzy soft sets (FSS), and fuzzy parameterized fuzzy soft sets (FPFS-sets) [2I], [22] have been used to model real-life problems in various fields such as engineering, medical science, quantum physics, environment, economics, and psychology, etc.

In 1965, Zadeh introduced the fuzzy set [32], which is used nowadays in almost all branches of science. It is basically a suitable modeling method as the crisp models lack clarification when dealing with issues in various scientific fields such as control engineering, artificial intelligence, decision theory, computer science, expert system, operational analysis, logic management science, robotics, and many others. In other words, this idea of the fuzzy set is used to resolve the

https://doi.org/10.52547/HATEF.JAHLA.2.4.6 
unpredictable problems that exist in models that reflect the phenomena of real life. Maiers and Sherif [1.9], in their index of fuzzy applications, listed more than twelve subject areas, including decision-making, engineering, economics, and operational analysis, based on the literature available on the subject. The concept of IFSs, which are generalizations of FS, is given in [4]. An IFS has both membership and membership degrees. Fuzzy parameterized fuzzy soft sets (FPFS-sets) [2I], [22] is the extension of an FS and soft set. The FPFS set preserves both elements and parameters to an appropriate membership degree. Ramot et, al., [28] introduced the notion of a complex CFS, the extension of the fuzzy set. A CFS membership function has all the values in the unit disk. A complex fuzzy set is used to describe two-dimensional phenomena and plays a significant role in periodic phenomena.

The notion of a neutrosophic set (NS) was given by Samarandache [30], [3T]. An NS is the generalization of a crisp set, FS and IFS. The membership of truth (TM) and falsehood (FM) and indeterminacy (IM) are independent in NS. The role of indeterminacy is very important for decision-making problems. In several areas, such as decision-making problems, medical diagnosis, image processing, educational problems, and dispute resolution, an NS and its extensions play a critical role.

Soft set theory has enhanced its potentiality since its introduction by Molodtsov [26]. On the basis of several soft-set operations introduced in [2], [23], [24], some additional properties and algebra can be found in [I]. The soft semirings [I:3] were introduced by Feng et, al., an adjustable approach to fuzzy soft sets-based decision making can be found in [1:2] by means of level soft sets. We can found some new ideas combined with rough sets and fuzzy sets in [14], [15]. Aygunoglu et, al., introduced the fuzzy soft groups [5].

By introducing a new idea called neutrosophic soft set, Maji [20] combined the notion of soft set and neutrosophic set together and offered an application of neutrosophic soft set to the problem of decision making. Recently, the properties and applications on the neutrosophic soft sets have been studied increasingly [6], [7], [10], [II]. Karaaslan [17] filled the gaps of the Maji's neutrosophic soft set [Z] definition and operations redefining the concept of neutrosophic soft set and operations between neutrosophic soft sets. Jha et, al., [16] proposed a model for stock trend prediction based on neutrosophic soft set (NSS). The bipolar neutrosophic soft sets notation, which combines soft sets and bipolar neutrosophic sets, was developed by Ali in [3]. They introduced a bipolar neutrosophic soft operator aggregation of a bipolar neutrosophic soft set and developed a bipolar neutrosophic soft set decision-making algorithm. Qamar et, al., [27] gave the notion of Q-neutrosophic-set aggregation operator and use it to develop an algorithm for using a Qneutrosophic soft set in decision-making issues. Saqlain et, al., [29] proposed an algorithm based on accuracy function under a neutrosophic soft set environment and used this proposed technique in decision-making problems. Moreover, Manna et, al., [25] offered a complex neutrosophic soft VIKOR approach to get a compromise optimal solution for single as well as multiple decision-maker based problems.

Çağman and Enginoğlu have implemented soft matrices and have investigated the different properties of the matrices. They have developed a soft max-min decision-making algorithm that can be used as a useful tool for uncertainty-related issues [8]. In addition, fuzzy soft matrices have been introduced along with the fs-max-min decision-making method, which can be used successfully in decision-making problems that contain ambiguity [9]. In fact, they gave a new direction for fundamental work as well as applications in the theory of soft sets and fuzzy soft sets. Madad et, al., [18] introduced complex fuzzy soft matrices and defined some new operations on these matrices. Moreover, they developed an algorithm using complex fuzzy soft matrices and applied it to a decision-making problem in signal processing. 
In decision-making problems, the use of soft matrices approaches is ubiquitous. The purpose of this article is two-fold. The first half aims to present the theoretical foundations of the neutrosophic soft metric matrix. These theoretical foundations contain the operations such as union, intersection, complement, difference, symmetric difference, etc on neutrosophic soft metric matrices. The second half aims to present these theoretical foundations and key techniques in decision-making problems and discuss the principle of the neutrosophic soft metric matrices in a coherent manner. The purpose of these innovative concepts is, to provide a new approach with useful mathematical tools to address the fundamental problem of decision-making. Neutrosophic soft metric matrix is the generalization of the fuzzy soft matrix. Although the degree of truth, false, and intermediate membership functions are implemented here, resulting in the better choice in decision-making problems. The generality of the neutrosophic soft metric matrix is given special importance, illustrating how many interesting optimization decision-making problems can be formulated as a problem of the neutrosophic soft metric matrix. These applied contexts provide solid evidence of the wide applications of the neutrosophic soft matrices approach to model and research decision-making problems. This article will stimulate the interest in the neutrosophic soft matrix and its application in decision-making problems.

In this paper, we introduce neutrosophic soft metric matrices and define some new operations on these matrices. Moreover, we develop an algorithm using neutrosophic soft metric matrices and apply it to a decision-making problem.

\section{Preliminaries}

We will discuss here the basic set-theoretic operations and laws of neutrosophic soft metric matrices and also discuss particular examples of these operations and laws.

Definition 2.1. [26] Let $U$ be an initial universe set and $E$ be a set of parameters. Let $P(U)$ denotes the power set of $U$. Consider a nonempty set $A, A \subset E$. A pair $(F, A)$ is called a soft set over $U$, where $F$ is a mapping given by $F: A \rightarrow P(U)$.

Definition 2.2. [30] A neutrosophic set $N$ on the universe of discourse $X$ is defined as $N=\{<$ $\left.x, T_{N}(x), I_{N}(x), F_{N}(x)>, x \in X\right\}$ where $\left.T, I, F: X \rightarrow\right]^{-} 0,1^{+}\left[\right.$and $^{-} 0 \leq T_{N}(x)+I_{N}(x)+F_{N}(x) \leq$ $3^{+}$.

From a philosophical point of view, the neutrosophic set takes the value from real standard or non-standard subsets of $]^{-} 0,1^{+}[$. But in real-life applications in scientific and engineering problems, it is difficult to use a neutrosophic set with the value from a real standard or a non-standard subset of $]^{-} 0,1^{+}[$. Hence we consider the neutrosophic set which takes the value from the subset of $[0,1]$.

Definition 2.3. [20] Let $U$ be an initial universe set and $E$ be a set of parameters. Consider $A \subset E$. Let $P(U)$ denotes the set of all neutrosophic sets of $U$. The collection $(F, A)$ is termed to be the soft neutrosophic set over $U$, where $F$ is a mapping given by $F: A \rightarrow P(U)$.

\section{$3 \quad$ Neutrosophic soft metric matrix theory}

In this section, we introduce a new concept of a neutrosophic soft metric matrix. 


\subsection{Neutrosophic soft metric matrix}

Let $U=\left\{h_{1}, h_{2}, \ldots, h_{n}\right\}$ be a universal set. Let $E=\left\{e_{1}, e_{2}, \ldots, e_{n}\right\}$ be the set of parameters. If $A \subset E$, then $\left(\epsilon_{\lambda_{i}}, A_{i}\right)$ represents a neutrosophic soft set over $U$, where $\epsilon_{\lambda}$ is a mapping given by $\epsilon_{\lambda}$ : $A \rightarrow P\left(\lambda_{U}\right)$, where $P\left(\lambda_{U}\right)$ denote the power sets of neutrosophic soft sets. Define $d_{h_{i}}: \epsilon_{\lambda} \rightarrow C^{U}$, where $C^{U}$ denotes the set of all fuzzy subsets and $P\left(C^{U}\right)$ denotes the power set of $C^{U}$. Then the neutrosophic soft set can be expressed in matrix form as $\left[X_{m \times n^{\prime}}\right]=\left[d_{h_{i}}\right]_{m \times n^{\prime}}$ for $i=1,2,3, \ldots, n$ where

$$
d_{h_{i}}\left(\epsilon_{\lambda}\left(e_{i}\right), \epsilon_{\lambda}\left(e_{j}\right)\right)=\left\{\begin{array}{rr}
d_{h_{i}}\left(\epsilon_{\lambda}\left(e_{i}\right), \epsilon_{\lambda}\left(e_{j}\right)\right) & \text { if } e_{i}, e_{j} \in A \\
0 & \text { otherwise }
\end{array} .\right.
$$

The columns $C_{1}, C_{2}, \ldots, C_{m}$ are labelled by the metrics $d_{h_{1}}, d_{h_{2}}, \ldots, d_{h_{m}}$.

\subsection{Representation of neutrosophic soft metric matrix}

The neutrosophic soft metric matrix is represented by the following:

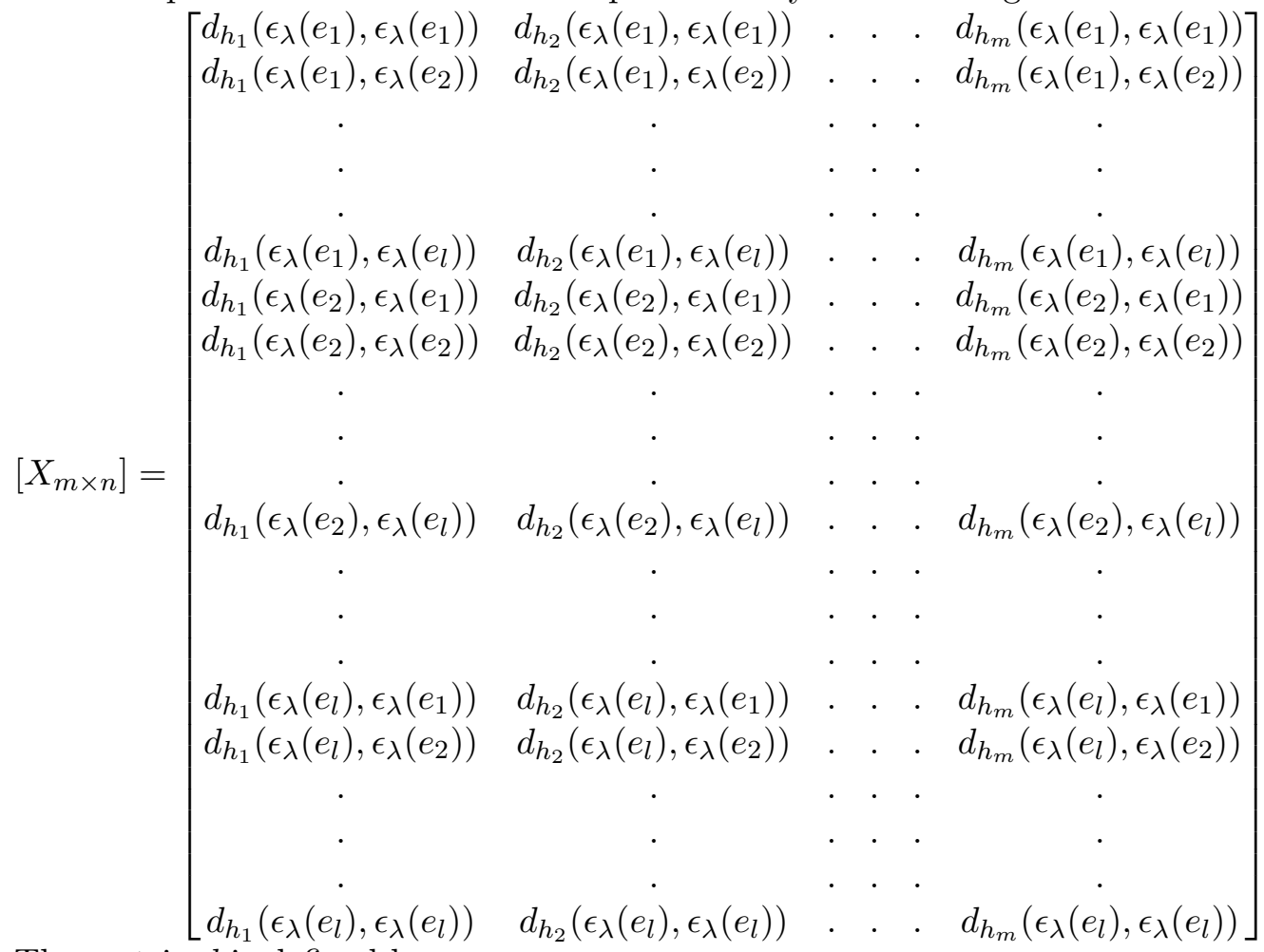

The metric $d$ is defined by

$$
d_{h_{i}}\left(\epsilon_{\lambda}\left(e_{k}\right), \epsilon_{\lambda}\left(e_{l}\right)\right)=\frac{1}{3}\left[\left|T_{h_{i}}^{e_{k}}-T_{h_{i}}^{e_{l}}\right|+\left|I_{h_{i}}^{e_{k}}-I_{h_{i}}^{e_{l}}\right|+\left|F_{h_{i}}^{e_{k}}-F_{h_{i}}^{e_{l}}\right|\right] \text { for all } i, j, k=1,2, \ldots, n .
$$

Example 3.1. Suppose there are three houses under consideration, namely the universes $U=$ $\left\{h_{1}, h_{2}, h_{3}\right\}$ and $E=\left\{e_{1}, e_{2}, e_{3}\right\}$ be the set of parameters where $e_{1}$ stands for "near to town", $e_{2}$ stands for "beautiful", $e_{3}$ stands for "expensive". Assume that $A=\left\{e_{1}, e_{3}\right\}$ be a subset of $E$. Suppose that

$$
\begin{aligned}
& \epsilon_{\lambda}\left(e_{1}\right)=\left\{\left\langle h_{1}, 0.5,0.6,0.9\right\rangle,\left\langle h_{2}, 0.2,0.5,0.3\right\rangle,\left\langle h_{3}, 0.8,0.4,0.9\right\rangle\right\}, \\
& \epsilon_{\lambda}\left(e_{3}\right)=\left\{\left\langle h_{1}, 0.2,0.5,0.8\right\rangle,\left\langle h_{2}, 0.3,0.9,0.5\right\rangle,\left\langle h_{3}, 0.4,0.6,0.6\right\rangle\right\} .
\end{aligned}
$$


For house $h_{1}$ we have

$d_{h_{i}}\left(\epsilon_{\lambda}\left(e_{2}\right), \epsilon_{\lambda}\left(e_{l}\right)\right)=0$ for $i=1,2,3$ and $l=1,3$.

$d_{h_{1}}\left(\epsilon_{\lambda}\left(e_{1}\right), \epsilon_{\lambda}\left(e_{1}\right)\right)=0, d_{h_{1}}\left(\epsilon_{\lambda}\left(e_{1}\right), \epsilon_{\lambda}\left(e_{2}\right)\right)=0, d_{h_{1}}\left(\epsilon_{\lambda}\left(e_{1}\right), \epsilon_{\lambda}\left(e_{3}\right)\right)=0.16$

$d_{h_{1}}\left(\epsilon_{\lambda}\left(e_{3}\right), \epsilon_{\lambda}\left(e_{1}\right)\right)=0.16, d_{h_{1}}\left(\epsilon_{\lambda}\left(e_{3}\right), \epsilon_{\lambda}\left(e_{2}\right)\right)=0, d_{h_{1}}\left(\epsilon_{\lambda}\left(e_{3}\right), \epsilon_{\lambda}\left(e_{3}\right)\right)=0$.

For house $h_{2}$, we have

$d_{h_{2}}\left(\epsilon_{\lambda}\left(e_{1}\right), \epsilon_{\lambda}\left(e_{1}\right)\right)=0, d_{h_{2}}\left(\epsilon_{\lambda}\left(e_{1}\right), \epsilon_{\lambda}\left(e_{2}\right)\right)=0, d_{h_{2}}\left(\epsilon_{\lambda}\left(e_{1}\right), \epsilon_{\lambda}\left(e_{3}\right)\right)=0.23$,

$d_{h_{2}}\left(\epsilon_{\lambda}\left(e_{3}\right), \epsilon_{\lambda}\left(e_{1}\right)\right)=0.23, d_{h_{2}}\left(\epsilon_{\lambda}\left(e_{3}\right), \epsilon_{\lambda}\left(e_{2}\right)\right)=0, d_{h_{2}}\left(\epsilon_{\lambda}\left(e_{3}\right), \epsilon_{\lambda}\left(e_{3}\right)\right)=0$.

For house $h_{3}$, we have

$d_{h_{3}}\left(\epsilon_{\lambda}\left(e_{1}\right), \epsilon_{\lambda}\left(e_{1}\right)\right)=0, d_{h_{3}}\left(\epsilon_{\lambda}\left(e_{1}\right), \epsilon_{\lambda}\left(e_{2}\right)\right)=0, d_{h_{3}}\left(\epsilon_{\lambda}\left(e_{1}\right), \epsilon_{\lambda}\left(e_{3}\right)\right)=0.3$,

$d_{h_{3}}\left(\epsilon_{\lambda}\left(e_{3}\right), \epsilon_{\lambda}\left(e_{1}\right)\right)=0.3, d_{h_{3}}\left(\epsilon_{\lambda}\left(e_{3}\right), \epsilon_{\lambda}\left(e_{2}\right)\right)=0, d_{h_{3}}\left(\epsilon_{\lambda}\left(e_{3}\right), \epsilon_{\lambda}\left(e_{3}\right)\right)=0$.

We would represent this neutrosophic soft set in matrix form as:

$$
\left[X_{9 \times 3}\right]=\left[\begin{array}{ccc}
0 & 0 & 0 \\
0 & 0 & 0 \\
0.16 & 0.23 & 0.3 \\
0 & 0 & 0 \\
0 & 0 & 0 \\
0 & 0 & 0 \\
0.16 & 0.23 & 0.3 \\
0 & 0 & 0 \\
0 & 0 & 0
\end{array}\right]
$$

Definition 3.2. Let $\left[X_{m \times n}\right]$ be a neutrosophic soft metric matrix. Then $\left[X_{m \times n}\right]$ is called neutrosophic zero soft metric matrix if $d_{h_{i}}\left(\epsilon_{\lambda}\left(e_{i}\right), \epsilon_{\lambda}\left(e_{j}\right)\right)=0$ for all $d_{h_{i}} \in\left[X_{m \times n}\right]$ and denoted by $\left[X_{m \times n}\right]=[0]$.

Example 3.3. The matrix $\left[X_{2 \times 4}\right]=\left[\begin{array}{ll}0 & 0 \\ 0 & 0 \\ 0 & 0 \\ 0 & 0\end{array}\right]$ is a neutrosophic zero soft metric matrix.

Definition 3.4. Let $\left[X_{m \times n}\right]$ and $\left[Y_{m \times n}\right]$ be two neutrosophic soft metric matrices. Then

$(i)$. [ $\left.X_{m \times n}\right]$ is a neutrosophic soft metric submatrices of $\left[Y_{m \times n}\right]$, is denoted by $\left[X_{m \times n}\right] \subseteq\left[Y_{m \times n}\right]$, if $d_{h_{i}} \leq d_{h_{i}}^{\prime}$ for all $d_{h_{i}} \in\left[X_{m \times n}\right]$ and $d_{h_{i}}^{\prime} \in\left[Y_{m \times n}\right]$.

$(i i) .\left[X_{m \times n}\right]$ is a proper neutrosophic soft metric submatrices of $\left[Y_{m \times n}\right]$, is denoted by $\left[X_{m \times n}\right] \subset$ $\left[Y_{m \times n}\right]$, if $d_{h_{i}} \leq d_{h_{i}}^{\prime}$ for all $d_{h_{i}} \in\left[X_{m \times n}\right]$ and $d_{h_{i}}^{\prime} \in\left[Y_{m \times n}\right]$, and for at least one term $d_{h_{i}}<d_{h_{i}}^{\prime}$.

(iii). $\left[X_{m \times n}\right]$ is an equal neutrosophic soft metric matrix of $\left[Y_{m \times n}\right]$, is denoted by $\left[X_{m \times n}\right]=$ $\left[Y_{m \times n}\right]$ if $d_{h_{i}}=d_{h_{i}}^{\prime}$ for all $d_{h_{i}} \in\left[X_{m \times n}\right]$ and $d_{h_{i}}^{\prime} \in\left[Y_{m \times n}\right]$.

Definition 3.5. Let $\left[X_{m \times n}\right]$ and $\left[Y_{m \times n}\right]$ be neutrosophic soft metric matrices. Then the neutrosophic soft metric matrices $\left[Z_{m \times n}\right]$ are called:

$(i)$. Union of $\left[X_{m \times n}\right]$ and $\left[Y_{m \times n}\right]$ is denoted by

$$
\left[X_{m \times n}\right] \cup\left[Y_{m \times n}\right]=\max \left\{d_{h_{i}}, d_{h_{i}}^{\prime}\right\},
$$

for all $d_{h_{i}} \in\left[X_{m \times n}\right]$ and $d_{h_{i}}^{\prime} \in\left[Y_{m \times n}\right]$.

(ii). Intersection of $\left[X_{m \times n}\right]$ and $\left[Y_{m \times n}\right]$ is denoted by

$$
\left[X_{m \times n}\right] \cap\left[Y_{m \times n}\right]=\min \left\{d_{h_{i}}, d_{h_{i}}^{\prime}\right\},
$$

for all $d_{h_{i}} \in\left[X_{m \times n}\right]$ and $d_{h_{i}}^{\prime} \in\left[Y_{m \times n}\right]$.

(iii). complement of $\left[X_{m \times n}\right]$ denoted by $\left[X_{m \times n}\right]^{\bullet}$ if $\left[X_{m \times n}\right]^{\bullet}=1-d_{h_{i}}$ for all $d_{h_{i}} \in\left[X_{m \times n}\right]$. 
Example 3.6. Assume that

$$
\begin{gathered}
{\left[X_{m \times n}\right]=\left[\begin{array}{cccc}
0 & 0 & 0 & 0 \\
0.6 & 0.9 & 1 & 0.4 \\
0.6 & 0.9 & 1 & 0.4 \\
0 & 0 & 0 & 0
\end{array}\right],\left[Y_{m \times n}\right]=\left[\begin{array}{cccc}
0 & 0 & 0 & 0 \\
0.3 & 1 & 0.8 & 0.5 \\
0.3 & 1 & 0.8 & 0.5 \\
0 & 0 & 0 & 0
\end{array}\right] . \text { Then }} \\
{\left[X_{m \times n}\right] \cup\left[Y_{m \times n}\right]=\left[\begin{array}{cccc}
0 & 0 & 0 & 0 \\
0.6 & 1 & 1 & 0.5 \\
0.6 & 1 & 1 & 0.5 \\
0 & 0 & 0 & 0
\end{array}\right] .}
\end{gathered}
$$

and

$$
\left[X_{m \times n}\right] \cap\left[Y_{m \times n}\right]=\left[\begin{array}{cccc}
0 & 0 & 0 & 0 \\
0.3 & 0.9 & 0.8 & 0.4 \\
0.3 & 0.9 & 0.8 & 0.4 \\
0 & 0 & 0 & 0
\end{array}\right] .
$$

Now, we have to find $\left[X_{m \times n}\right]^{\bullet}$, that is,

$$
\left[X_{m \times n}\right]^{\bullet}=\left[\begin{array}{cccc}
1 & 1 & 1 & 1 \\
0.4 & 0.1 & 0 & 0.6 \\
0.4 & 0.1 & 0 & 0.6 \\
1 & 1 & 1 & 1
\end{array}\right]
$$

Definition 3.7. Let $\left[X_{m \times n}\right]$ and $\left[Y_{m \times n}\right]$ be neutrosophic soft metric matrices. Then $\left[X_{m \times n}\right]$ and $\left[Y_{m \times n}\right]$ are disjoint if $\left[X_{m \times n}\right] \cap\left[Y_{m \times n}\right]=[0]$ for all $m$ and $n$.

Definition 3.8. Let $\left[X_{m \times n}\right]$ and $\left[Y_{m \times n}\right]$ be neutrosophic soft metric matrices. Then the difference of these two neutrosophic soft metric matrices is defined by

$$
\left[X_{m \times n}\right] /\left[Y_{m \times n}\right]=d_{h_{i}}-d_{h_{i}}^{\prime}
$$

for all $d_{h_{i}} \in\left[X_{m \times n}\right]$ and $d_{h_{i}}^{\prime} \in\left[Y_{m \times n}\right]$.

Definition 3.9. Let $\left[X_{m \times n}\right]$ and $\left[Y_{m \times n}\right]$ be two neutrosophic soft metric matrices. Then the symmetric difference of these two neutrosophic soft metric matrices denoted by $\left[X_{m \times n}\right] \Delta\left[Y_{m \times n}\right]$ and is defined as:

$$
\begin{aligned}
{\left[X_{m \times n}\right] \Delta\left[Y_{m \times n}\right] } & =\left\{\left[X_{m \times n}\right] \cup\left[Y_{m \times n}\right]\right\}-\left\{\left[X_{m \times n}\right] \cap\left[Y_{m \times n}\right]\right\} \\
& =\max \left\{d_{h_{i}}, d_{h_{i}}^{\prime}\right\}-\min \left\{d_{h_{i}}, d_{h_{i}}^{\prime}\right\}
\end{aligned}
$$

for all $d_{h_{i}} \in\left[X_{m \times n}\right]$ and $d_{h_{i}}^{\prime} \in\left[Y_{m \times n}\right]$.

\section{Main results}

Proposition 4.1. Let $\left[X_{m \times n}\right]$ be a neutrosophic soft metric matrix. Then

(i) $\left(\left[X_{m \times n}\right]^{\bullet}\right)^{\bullet}=\left[X_{m \times n}\right]$.

$($ ii $) \cdot[0]^{\bullet}=[1]$.

Proof. It can be proved easily. 
Proposition 4.2. Let $\left[X_{m \times n}\right]$ and $\left[Y_{m \times n}\right]$ be neutrosophic soft metric matrices. Then De-Margon Law are valid.

(i). $\left(\left[X_{m \times n}\right] \cup\left[Y_{m \times n}\right]\right)^{\bullet}=\left[X_{m \times n}\right]^{\bullet} \cap\left[Y_{m \times n}\right]^{\bullet}$.

(ii). $\left(\left[X_{m \times n}\right] \cap\left[Y_{m \times n}\right]\right)^{\bullet}=\left[X_{m \times n}\right]^{\bullet} \cup\left[Y_{m \times n}\right]^{\bullet}$.

Proof. (i). For all $m, n$

$$
\begin{aligned}
\left(\left[X_{m \times n}\right] \cup\left[Y_{m \times n}\right]\right)^{\bullet} & =\left(\max \left\{d_{h_{i}}, d_{h_{i}}^{\prime}\right\}\right)^{\bullet}=1-\max \left\{d_{h_{i}}, d_{h_{i}}^{\prime}\right\} \\
& =\min \left\{1-d_{h_{i}}, 1-d_{h_{i}}^{\prime}\right\}=\left[X_{m \times n}\right]^{\bullet} \cap\left[Y_{m \times n}\right]^{\bullet} .
\end{aligned}
$$

(ii). It can be proved similarly.

Proposition 4.3. If $\left[X_{m \times n}\right],\left[Y_{m \times n}\right]$ and $\left[Z_{m \times n}\right]$ are neutrosophic soft metric matrices, then

(i). $\left[X_{m \times n}\right]=\left[Y_{m \times n}\right]$ and $\left[Y_{m \times n}\right]=\left[Z_{m \times n}\right] \Longrightarrow\left[X_{m \times n}\right]=\left[Z_{m \times n}\right]$.

(ii). $\left[X_{m \times n}\right] \subseteq\left[Y_{m \times n}\right]$ and $\left[Y_{m \times n}\right] \subseteq\left[X_{m \times n}\right] \Longleftrightarrow\left[X_{m \times n}\right]=\left[Y_{m \times n}\right]$.

Proof. $(i)$. Assume that $\left[X_{m \times n}\right]=\left[Y_{m \times n}\right]$ and $\left[Y_{m \times n}\right]=\left[Z_{m \times n}\right]$. Then

$$
d_{h_{i}}=d_{h_{i}}^{\prime}
$$

and

$$
d_{h_{i}}^{\prime}=d_{h_{i}}^{\prime \prime},
$$

for $d_{h_{i}} \in\left[X_{m \times n}\right], d_{h_{i}}^{\prime} \in\left[Y_{m \times n}\right]$ and $d_{h_{i}}^{\prime \prime} \in\left[Z_{m \times n}\right]$. By transitive property, we have

$$
d_{h_{i}}=d_{h_{i}}^{\prime \prime}
$$

From (3), we have

$$
\left[X_{m \times n}\right]=\left[Y_{m \times n}\right] .
$$

(ii). Assume that $\left[X_{m \times n}\right] \subseteq\left[Y_{m \times n}\right]$ and $\left[Y_{m \times n}\right] \subseteq\left[X_{m \times n}\right]$. Then

$$
d_{h_{i}} \leq d_{h_{i}}^{\prime}
$$

and

$$
d_{h_{i}}^{\prime} \leq d_{h_{i}}
$$

for $d_{h_{i}} \in\left[X_{m \times n}\right]$ and $d_{h_{i}}^{\prime} \in\left[Y_{m \times n}\right]$. From (3) and (4), we have

$$
d_{h_{i}}=d_{h_{i}}^{\prime} .
$$

From (3), we have

$$
\left[X_{m \times n}\right]=\left[Y_{m \times n}\right] .
$$

Conversely assume that $\left[X_{m \times n}\right]=\left[Y_{m \times n}\right]$. Then for all $d_{h_{i}} \in\left[X_{m \times n}\right]$ and $d_{h_{i}}^{\prime} \in\left[Y_{m \times n}\right]$, we have

$$
d_{h_{i}}=d_{h_{i}}^{\prime} .
$$

This implies that $d_{h_{i}} \leq d_{h_{i}}^{\prime}$ and $d_{h_{i}}^{\prime} \leq d_{h_{i}}$ and hence $\left[X_{m \times n}\right] \subseteq\left[Y_{m \times n}\right]$ and $\left[Y_{m \times n}\right] \subseteq\left[X_{m \times n}\right]$.

Proposition 4.4. If $\left[X_{m \times n}\right],\left[Y_{m \times n}\right]$ and $\left[Z_{m \times n}\right]$ are neutrosophic soft metric matrices, then

(i). $\left[X_{m \times n}\right] \subseteq\left[Y_{m \times n}\right]$ and $\left[Y_{m \times n}\right] \subseteq\left[Z_{m \times n}\right] \Longrightarrow\left[X_{m \times n}\right] \subseteq\left[Z_{m \times n}\right]$.

(ii). $\left[X_{m \times n}\right] \subseteq\left[Y_{m \times n}\right] \Longleftrightarrow\left[X_{m \times n}\right] \cap\left[Y_{m \times n}\right]=\left[X_{m \times n}\right]$.

(iii). $\left[X_{m \times n}\right] \subseteq\left[Y_{m \times n}\right] \Longleftrightarrow\left[X_{m \times n}\right] \cup\left[Y_{m \times n}\right]=\left[Y_{m \times n}\right]$. 
Proof. (i). Assume that $\left[X_{m \times n}\right] \subseteq\left[Y_{m \times n}\right]$ and $\left[Y_{m \times n}\right] \subseteq\left[Z_{m \times n}\right]$. Then

$$
d_{h_{i}} \leq d_{h_{i}}^{\prime}
$$

and

$$
d_{h_{i}}^{\prime} \leq d_{h_{i}}^{\prime \prime}
$$

for all $d_{h_{i}} \in\left[X_{m \times n}\right], d_{h_{i}}^{\prime} \in\left[Y_{m \times n}\right]$ and $d_{h_{i}}^{\prime \prime} \in\left[Z_{m \times n}\right]$. From (1) and (2), we have $d_{h_{i}} \leq d_{h_{i}}^{\prime \prime}$ and hence $\left[X_{m \times n}\right] \subseteq\left[Z_{m \times n}\right]$.

(ii). Assume that $\left[X_{m \times n}\right] \subseteq\left[Y_{m \times n}\right]$. Then for all $d_{h_{i}} \in\left[X_{m \times n}\right], d_{h_{i}}^{\prime} \in\left[Y_{m \times n}\right]$, we have

$$
d_{h_{i}} \leq d_{h_{i}}^{\prime}
$$

Now,

$$
\left[X_{m \times n}\right] \cap\left[Y_{m \times n}\right]=\min \left\{d_{h_{i}}, d_{h_{i}}^{\prime}\right\}=d_{h_{i}} .
$$

Thus we have

$$
\left[X_{m \times n}\right] \cap\left[Y_{m \times n}\right]=\left[X_{m \times n}\right]
$$

Conversely assume that

$$
\left[X_{m \times n}\right] \cap\left[Y_{m \times n}\right]=\left[X_{m \times n}\right] .
$$

From (4), we have $d_{h_{i}} \leq d_{h_{i}}^{\prime}$ for all $d_{h_{i}} \in\left[X_{m \times n}\right]$ and $d_{h_{i}}^{\prime} \in\left[Y_{m \times n}\right]$ and hence $\left[X_{m \times n}\right] \subseteq\left[Y_{m \times n}\right]$. (iii). Assume that $\left[X_{m \times n}\right] \subseteq\left[Y_{m \times n}\right]$. Then for all $d_{h_{i}} \in\left[X_{m \times n}\right], d_{h_{i}}^{\prime} \in\left[Y_{m \times n}\right]$, we have

$$
d_{h_{i}} \leq d_{h_{i}}^{\prime}
$$

Now,

$$
\left[X_{m \times n}\right] \cup\left[Y_{m \times n}\right]=\max \left\{d_{h_{i}}, d_{h_{i}}^{\prime}\right\}=d_{h_{i}}^{\prime} .
$$

Thus

$$
\left[X_{m \times n}\right] \cup\left[Y_{m \times n}\right]=\left[Y_{m \times n}\right] .
$$

Conversely assume that

$$
\left[X_{m \times n}\right] \cup\left[Y_{m \times n}\right]=\left[Y_{m \times n}\right] .
$$

From (6), we have $d_{h_{i}} \leq d_{h_{i}}^{\prime}$ for all $d_{h_{i}} \in\left[X_{m \times n}\right]$ and $d_{h_{i}}^{\prime} \in\left[Y_{m \times n}\right]$ and so $\left[X_{m \times n}\right] \subseteq\left[Y_{m \times n}\right]$.

Proposition 4.5. If $\left[X_{m \times n}\right]$ and $\left[Y_{m \times n}\right]$ are neutrosophic soft metric matrices, then

(i). $\left[X_{m \times n}\right] \cup\left[Y_{m \times n}\right]=\left[Y_{m \times n}\right] \cup\left[X_{m \times n}\right]$.

(ii). $\left[X_{m \times n}\right] \cap\left[Y_{m \times n}\right]=\left[Y_{m \times n}\right] \cap\left[X_{m \times n}\right]$.

Proof. To prove $(i)$, two cases arise here.

Case 1. For all $d_{h_{i}} \in\left[X_{m \times n}\right]$ and $d_{h_{i}}^{\prime} \in\left[Y_{m \times n}\right]$ such that $d_{h_{i}} \leq d_{h_{i}}^{\prime}$. Then

$$
\left[X_{m \times n}\right] \cup\left[Y_{m \times n}\right]=\max \left\{d_{h_{i}}, d_{h_{i}}^{\prime}\right\}=d_{h_{i}}^{\prime} .
$$

Also,

$$
\left[Y_{m \times n}\right] \cup\left[X_{m \times n}\right]=\max \left\{d_{h_{i}}^{\prime}, d_{h_{i}}\right\}=d_{h_{i}}^{\prime} .
$$

From (1) and (2), we have

$$
\left[X_{m \times n}\right] \cup\left[Y_{m \times n}\right]=\left[Y_{m \times n}\right] \cup\left[X_{m \times n}\right] .
$$


Case 2. For all $d_{h_{i}} \in\left[X_{m \times n}\right]$ and $d_{h_{i}}^{\prime} \in\left[Y_{m \times n}\right]$ such that $d_{h_{i}}^{\prime} \leq d_{h_{i}}$. Then

$$
\left[X_{m \times n}\right] \cup\left[Y_{m \times n}\right]=\max \left\{d_{h_{i}}, d_{h_{i}}^{\prime}\right\}=d_{h_{i}} .
$$

Also,

$$
\left[Y_{m \times n}\right] \cup\left[X_{m \times n}\right]=\max \left\{d_{h_{i}}^{\prime}, d_{h_{i}}\right\}=d_{h_{i}} .
$$

From (3) and (4), we have

$$
\left[X_{m \times n}\right] \cup\left[Y_{m \times n}\right]=\left[Y_{m \times n}\right] \cup\left[X_{m \times n}\right] .
$$

(ii). It can be proved similarly.

Theorem 4.6. If $\left[X_{m \times n}\right],\left[Y_{m \times n}\right]$ and $\left[Z_{m \times n}\right]$ are neutrosophic soft metric matrices, then (i). $\left(\left[X_{m \times n}\right] \cup\left[Y_{m \times n}\right]\right) \cup\left[Z_{m \times n}\right]=\left[X_{m \times n}\right] \cup\left(\left[Y_{m \times n}\right] \cup\left[Z_{m \times n}\right]\right)$.

(ii). $\left(\left[X_{m \times n}\right] \cap\left[Y_{m \times n}\right]\right) \cap\left[Z_{m \times n}\right]=\left[X_{m \times n}\right] \cap\left(\left[Y_{m \times n}\right] \cap\left[Z_{m \times n}\right]\right)$.

Proof. To prove $(i)$, four cases arise here.

Case 3. If $d_{h_{i}} \leq d_{h_{i}}^{\prime} \leq d_{h_{i}}^{\prime \prime}$ for all $d_{h_{i}} \in\left[X_{m \times n}\right], d_{h_{i}}^{\prime} \in\left[Y_{m \times n}\right]$ and $d_{h_{i}}^{\prime \prime} \in\left[Z_{m \times n}\right]$, then

$$
\begin{gathered}
\left(\left[X_{m \times n}\right] \cup\left[Y_{m \times n}\right]\right) \cup\left[Z_{m \times n}\right]=\max \left\{\max \left\{d_{h_{i}}, d_{h_{i}}^{\prime}\right\}, d_{h_{i}}^{\prime \prime}\right\}=\max \left\{d_{h_{i}}^{\prime}, d_{h_{i}}^{\prime \prime}\right\} . \\
\left(\left[X_{m \times n}\right] \cup\left[Y_{m \times n}\right]\right) \cup\left[Z_{m \times n}\right]=d_{h_{i}}^{\prime \prime} . \\
{\left[X_{m \times n}\right] \cup\left(\left[Y_{m \times n}\right] \cup\left[Z_{m \times n}\right]\right)=\max \left\{d_{h_{i}}, \max \left\{d_{h_{i}}^{\prime}, d_{h_{i}}^{\prime \prime}\right\}\right\}=\max \left\{d_{h_{i}}, d_{h_{i}}^{\prime \prime}\right\} .} \\
{\left[X_{m \times n}\right] \cup\left(\left[Y_{m \times n}\right] \cup\left[Z_{m \times n}\right]\right)=d_{h_{i}}^{\prime \prime} .}
\end{gathered}
$$

From (1) and (2), we have

$$
\left(\left[X_{m \times n}\right] \cup\left[Y_{m \times n}\right]\right) \cup\left[Z_{m \times n}\right]=\left[X_{m \times n}\right] \cup\left(\left[Y_{m \times n}\right] \cup\left[Z_{m \times n}\right]\right) .
$$

Case 4. If $d_{h_{i}}^{\prime \prime} \leq d_{h_{i}}^{\prime} \leq d_{h_{i}}$ for all $d_{h_{i}} \in\left[X_{m \times n}\right], d_{h_{i}}^{\prime} \in\left[Y_{m \times n}\right]$ and $d_{h_{i}}^{\prime \prime} \in\left[Z_{m \times n}\right]$, then

$$
\begin{gathered}
\left(\left[X_{m \times n}\right] \cup\left[Y_{m \times n}\right]\right) \cup\left[Z_{m \times n}\right]=\max \left\{\max \left\{d_{h_{i}}, d_{h_{i}}^{\prime}\right\}, d_{h_{i}}^{\prime \prime}\right\}=\max \left\{d_{h_{i}}, d_{h_{i}}^{\prime \prime}\right\} . \\
\left(\left[X_{m \times n}\right] \cup\left[Y_{m \times n}\right]\right) \cup\left[Z_{m \times n}\right]=d_{h_{i}} . \\
{\left[X_{m \times n}\right] \cup\left(\left[Y_{m \times n}\right] \cup\left[Z_{m \times n}\right]\right)=\max \left\{d_{h_{i}}, \max \left\{d_{h_{i}}^{\prime}, d_{h_{i}}^{\prime \prime}\right\}\right\}=\max \left\{d_{h_{i}}, d_{h_{i}}^{\prime}\right\} .} \\
{\left[X_{m \times n}\right] \cup\left(\left[Y_{m \times n}\right] \cup\left[Z_{m \times n}\right]\right)=d_{h_{i}} .}
\end{gathered}
$$

From (3) and (4), we have

$$
\left(\left[X_{m \times n}\right] \cup\left[Y_{m \times n}\right]\right) \cup\left[Z_{m \times n}\right]=\left[X_{m \times n}\right] \cup\left(\left[Y_{m \times n}\right] \cup\left[Z_{m \times n}\right]\right) .
$$

Case 5. If $d_{h_{i}}^{\prime} \leq d_{h_{i}}^{\prime \prime} \leq d_{h_{i}}$ for all $d_{h_{i}} \in\left[X_{m \times n}\right], d_{h_{i}}^{\prime} \in\left[Y_{m \times n}\right]$ and $d_{h_{i}}^{\prime \prime} \in\left[Z_{m \times n}\right]$, then

$$
\left(\left[X_{m \times n}\right] \cup\left[Y_{m \times n}\right]\right) \cup\left[Z_{m \times n}\right]=\max \left\{\max \left\{d_{h_{i}}, d_{h_{i}}^{\prime}\right\}, d_{h_{i}}^{\prime \prime}\right\}=\max \left\{d_{h_{i}}, d_{h_{i}}^{\prime \prime}\right\} .
$$




$$
\begin{gathered}
\left(\left[X_{m \times n}\right] \cup\left[Y_{m \times n}\right]\right) \cup\left[Z_{m \times n}\right]=d_{h_{i}} . \\
{\left[X_{m \times n}\right] \cup\left(\left[Y_{m \times n}\right] \cup\left[Z_{m \times n}\right]\right)=\max \left\{d_{h_{i}}, \max \left\{d_{h_{i}}^{\prime}, d_{h_{i}}^{\prime \prime}\right\}\right\}=\max \left\{d_{h_{i}}, d_{h_{i}}^{\prime \prime}\right\} .} \\
{\left[X_{m \times n}\right] \cup\left(\left[Y_{m \times n}\right] \cup\left[Z_{m \times n}\right]\right)=d_{h_{i}} .}
\end{gathered}
$$

From (5) and (6), we have

$$
\left(\left[X_{m \times n}\right] \cup\left[Y_{m \times n}\right]\right) \cup\left[Z_{m \times n}\right]=\left[X_{m \times n}\right] \cup\left(\left[Y_{m \times n}\right] \cup\left[Z_{m \times n}\right]\right) .
$$

Case 6. If $d_{h_{i}} \leq d_{h_{i}}^{\prime \prime} \leq d_{h_{i}}^{\prime}$ for all $d_{h_{i}} \in\left[X_{m \times n}\right], d_{h_{i}}^{\prime} \in\left[Y_{m \times n}\right]$ and $d_{h_{i}}^{\prime \prime} \in\left[Z_{m \times n}\right]$, then

$$
\begin{gathered}
\left(\left[X_{m \times n}\right] \cup\left[Y_{m \times n}\right]\right) \cup\left[Z_{m \times n}\right]=\max \left\{\max \left\{d_{h_{i}}, d_{h_{i}}^{\prime}\right\}, d_{h_{i}}^{\prime \prime}\right\}=\max \left\{d_{h_{i}}^{\prime}, d_{h_{i}}^{\prime \prime}\right\} . \\
\left(\left[X_{m \times n}\right] \cup\left[Y_{m \times n}\right]\right) \cup\left[Z_{m \times n}\right]=d_{h_{i}}^{\prime} . \\
{\left[X_{m \times n}\right] \cup\left(\left[Y_{m \times n}\right] \cup\left[Z_{m \times n}\right]\right)=\max \left\{d_{h_{i}}, \max \left\{d_{h_{i}}^{\prime}, d_{h_{i}}^{\prime \prime}\right\}\right\}=\max \left\{d_{h_{i}}, d_{h_{i}}^{\prime}\right\} .} \\
{\left[X_{m \times n}\right] \cup\left(\left[Y_{m \times n}\right] \cup\left[Z_{m \times n}\right]\right)=d_{h_{i}}^{\prime} .}
\end{gathered}
$$

From (7) and (8), we have

$$
\left(\left[X_{m \times n}\right] \cup\left[Y_{m \times n}\right]\right) \cup\left[Z_{m \times n}\right]=\left[X_{m \times n}\right] \cup\left(\left[Y_{m \times n}\right] \cup\left[Z_{m \times n}\right]\right) .
$$

(ii). It can be proved similarly.

Theorem 4.7. If $\left[X_{m \times n}\right],\left[Y_{m \times n}\right]$ and $\left[Z_{m \times n}\right]$ are neutrosophic soft metric matrices then

(i). $\left[X_{m \times n}\right] \cup\left(\left[Y_{m \times n}\right] \cap\left[Z_{m \times n}\right]\right)=\left(\left[X_{m \times n}\right] \cup\left[Y_{m \times n}\right]\right) \cap\left(\left[X_{m \times n}\right] \cup\left[Z_{m \times n}\right]\right)$.

(ii). $\left[X_{m \times n}\right] \cap\left(\left[Y_{m \times n}\right] \cup\left[Z_{m \times n}\right]\right)=\left(\left[X_{m \times n}\right] \cap\left(\left[Y_{m \times n}\right]\right) \cup\left(\left[X_{m \times n}\right] \cap\left[Z_{m \times n}\right]\right)\right.$.

Proof. To prove $(i)$, four cases arise here.

Case 7. If $d_{h_{i}} \leq d_{h_{i}}^{\prime} \leq d_{h_{i}}^{\prime \prime}$ for all $d_{h_{i}} \in\left[X_{m \times n}\right], d_{h_{i}}^{\prime} \in\left[Y_{m \times n}\right]$ and $d_{h_{i}}^{\prime \prime} \in\left[Z_{m \times n}\right]$. Then

$$
\begin{gathered}
{\left[X_{m \times n}\right] \cup\left(\left[Y_{m \times n}\right] \cap\left[Z_{m \times n}\right]\right)=\max \left\{d_{h_{i}}, \min \left\{d_{h_{i}}^{\prime}, d_{h_{i}}^{\prime \prime}\right\}\right\}=\max \left\{d_{h_{i}}, d_{h_{i}}^{\prime}\right\} .} \\
{\left[X_{m \times n}\right] \cup\left(\left[Y_{m \times n}\right] \cap\left[Z_{m \times n}\right]\right)=d_{h_{i}}^{\prime} .} \\
\left(\left[X_{m \times n}\right] \cup\left[Y_{m \times n}\right]\right) \cap\left(\left[X_{m \times n}\right] \cup\left[Z_{m \times n}\right]\right)=\min \left\{\max \left\{d_{h_{i}}, d_{h_{i}}^{\prime}\right\}, \max \left\{d_{h_{i}}, d_{h_{i}}^{\prime \prime}\right\}\right\}=\min \left\{d_{h_{i}}^{\prime}, d_{h_{i}}^{\prime \prime}\right\} . \\
\left(\left[X_{m \times n}\right] \cup\left[Y_{m \times n}\right]\right) \cap\left(\left[X_{m \times n}\right] \cup\left[Z_{m \times n}\right]\right)=d_{h_{i}}^{\prime} .
\end{gathered}
$$

From (1) and (2), we have

$$
\left[X_{m \times n}\right] \cup\left(\left[Y_{m \times n}\right] \cap\left[Z_{m \times n}\right]\right)=\left(\left[X_{m \times n}\right] \cup\left[Y_{m \times n}\right]\right) \cap\left(\left[X_{m \times n}\right] \cup\left[Z_{m \times n}\right]\right) .
$$


Case 8. If $d_{h_{i}}^{\prime \prime} \leq d_{h_{i}}^{\prime} \leq d_{h_{i}}$ for all $d_{h_{i}} \in\left[X_{m \times n}\right], d_{h_{i}}^{\prime} \in\left[Y_{m \times n}\right]$ and $d_{h_{i}}^{\prime \prime} \in\left[Z_{m \times n}\right]$. Then

$$
\begin{gathered}
{\left[X_{m \times n}\right] \cup\left(\left[Y_{m \times n}\right] \cap\left[Z_{m \times n}\right]\right)=\max \left\{d_{h_{i}}, \min \left\{d_{h_{i}}^{\prime}, d_{h_{i}}^{\prime \prime}\right\}\right\}=\max \left\{d_{h_{i}}, d_{h_{i}}^{\prime \prime}\right\} .} \\
{\left[X_{m \times n}\right] \cup\left(\left[Y_{m \times n}\right] \cap\left[Z_{m \times n}\right]\right)=d_{h_{i}}} \\
\left(\left[X_{m \times n}\right] \cup\left[Y_{m \times n}\right]\right) \cap\left(\left[X_{m \times n}\right] \cup\left[Z_{m \times n}\right]\right)=\min \left\{\max \left\{d_{h_{i}}, d_{h_{i}}^{\prime}\right\}, \max \left\{d_{h_{i}}, d_{h_{i}}^{\prime \prime}\right\}\right\}=\min \left\{d_{h_{i}}, d_{h_{i}}\right\} . \\
\left(\left[X_{m \times n}\right] \cup\left[Y_{m \times n}\right]\right) \cap\left(\left[X_{m \times n}\right] \cup\left[Z_{m \times n}\right]\right)=d_{h_{i}} .
\end{gathered}
$$

From (3) and (4), we have

$$
\left[X_{m \times n}\right] \cup\left(\left[Y_{m \times n}\right] \cap\left[Z_{m \times n}\right]\right)=\left(\left[X_{m \times n}\right] \cup\left[Y_{m \times n}\right]\right) \cap\left(\left[X_{m \times n}\right] \cup\left[Z_{m \times n}\right]\right) .
$$

Case 9. If $d_{h_{i}}^{\prime} \leq d_{h_{i}}^{\prime \prime} \leq d_{h_{i}}$ for all $d_{h_{i}} \in\left[X_{m \times n}\right], d_{h_{i}}^{\prime} \in\left[Y_{m \times n}\right]$ and $d_{h_{i}}^{\prime \prime} \in\left[Z_{m \times n}\right]$. Then

$$
\begin{gathered}
{\left[X_{m \times n}\right] \cup\left(\left[Y_{m \times n}\right] \cap\left[Z_{m \times n}\right]\right)=\max \left\{d_{h_{i}}, \min \left\{d_{h_{i}}^{\prime}, d_{h_{i}}^{\prime \prime}\right\}\right\}=\max \left\{d_{h_{i}}, d_{h_{i}}^{\prime}\right\} .} \\
{\left[X_{m \times n}\right] \cup\left(\left[Y_{m \times n}\right] \cap\left[Z_{m \times n}\right]\right)=d_{h_{i}} .} \\
\left(\left[X_{m \times n}\right] \cup\left[Y_{m \times n}\right]\right) \cap\left(\left[X_{m \times n}\right] \cup\left[Z_{m \times n}\right]\right)=\min \left\{\max \left\{d_{h_{i}}, d_{h_{i}}^{\prime}\right\}, \max \left\{d_{h_{i}}, d_{h_{i}}^{\prime \prime}\right\}\right\}=\min \left\{d_{h_{i}}, d_{h_{i}}\right\} . \\
\left(\left[X_{m \times n}\right] \cup\left[Y_{m \times n}\right]\right) \cap\left(\left[X_{m \times n}\right] \cup\left[Z_{m \times n}\right]\right)=d_{h_{i}} .
\end{gathered}
$$

From (5) and (6), we have

$$
\left[X_{m \times n}\right] \cup\left(\left[Y_{m \times n}\right] \cap\left[Z_{m \times n}\right]\right)=\left(\left[X_{m \times n}\right] \cup\left[Y_{m \times n}\right]\right) \cap\left(\left[X_{m \times n}\right] \cup\left[Z_{m \times n}\right]\right) .
$$

Case 10. If $d_{h_{i}} \leq d_{h_{i}}^{\prime \prime} \leq d_{h_{i}}^{\prime}$ for all $d_{h_{i}} \in\left[X_{m \times n}\right], d_{h_{i}}^{\prime} \in\left[Y_{m \times n}\right]$ and $d_{h_{i}}^{\prime \prime} \in\left[Z_{m \times n}\right]$. Then

$$
\begin{gathered}
{\left[X_{m \times n}\right] \cup\left(\left[Y_{m \times n}\right] \cap\left[Z_{m \times n}\right]\right)=\max \left\{d_{h_{i}}, \min \left\{d_{h_{i}}^{\prime}, d_{h_{i}}^{\prime \prime}\right\}\right\}=\max \left\{d_{h_{i}}, d_{h_{i}}^{\prime \prime}\right\} .} \\
{\left[X_{m \times n}\right] \cup\left(\left[Y_{m \times n}\right] \cap\left[Z_{m \times n}\right]\right)=d_{h_{i}}^{\prime \prime}} \\
\left(\left[X_{m \times n}\right] \cup\left[Y_{m \times n}\right]\right) \cap\left(\left[X_{m \times n}\right] \cup\left[Z_{m \times n}\right]\right)=\min \left\{\max \left\{d_{h_{i}}, d_{h_{i}}^{\prime}\right\}, \max \left\{d_{h_{i}}, d_{h_{i}}^{\prime \prime}\right\}\right\}=\min \left\{d_{h_{i}}^{\prime}, d_{h_{i}}^{\prime \prime}\right\} . \\
\left(\left[X_{m \times n}\right] \cup\left[Y_{m \times n}\right]\right) \cap\left(\left[X_{m \times n}\right] \cup\left[Z_{m \times n}\right]\right)=d_{h_{i}}^{\prime \prime} .
\end{gathered}
$$

From (7) and (8), we have

$$
\left[X_{m \times n}\right] \cup\left(\left[Y_{m \times n}\right] \cap\left[Z_{m \times n}\right]\right)=\left(\left[X_{m \times n}\right] \cup\left[Y_{m \times n}\right]\right) \cap\left(\left[X_{m \times n}\right] \cup\left[Z_{m \times n}\right]\right) .
$$

(ii). It can be proved similarly.

Theorem 4.8. Let $\chi$ be any collection of neutrosophic soft metric matrices of the same order and " " be a relation defined as " $\left[X_{m \times n}\right] \sim\left[Y_{m \times n}\right]$ if $d_{h_{i}} \leq d_{h_{i}}^{\prime} "$ for all $d_{h_{i}} \in\left[X_{m \times n}\right]$ and $d_{h_{i}}^{\prime} \in\left[Y_{m \times n}\right]$. Then the relation $" \sim$ is an order relation. 
Theorem 4.9. For any three neutrosophic soft metric matrices $\left[X_{m \times n}\right],\left[Y_{m \times n}\right]$ and $\left[Z_{m \times n}\right]$, the union is not distributive over symmetric difference $\Delta$.

Proof. For any three neutrosophic soft metric matrices $\left[X_{m \times n}\right],\left[Y_{m \times n}\right]$ and $\left[Z_{m \times n}\right]$, we have to show that

$$
\left[X_{m \times n}\right] \cup\left\{\left[Y_{m \times n}\right] \Delta\left[Z_{m \times n}\right]\right\} \neq\left\{\left[X_{m \times n}\right] \cup\left[Y_{m \times n}\right]\right\} \Delta\left\{\left[X_{m \times n}\right] \cup\left[Z_{m \times n}\right]\right\} .
$$

To prove the above we assume that

$$
d_{h_{i}} \leq d_{h_{i}}^{\prime} \leq d_{h_{i}}^{\prime \prime},
$$

for all $d_{h_{i}} \in\left[X_{m \times n}\right], d_{h_{i}}^{\prime} \in\left[Y_{m \times n}\right]$ and $d_{h_{i}}^{\prime \prime} \in\left[Z_{m \times n}\right]$. Now

$$
\left[Y_{m \times n}\right] \Delta\left[Z_{m \times n}\right]=\max \left\{d_{h_{i}}^{\prime}, d_{h_{i}}^{\prime \prime}\right\}-\min \left\{d_{h_{i}}^{\prime}, d_{h_{i}}^{\prime \prime}\right\}=d_{h_{i}}^{\prime \prime}-d_{h_{i}}^{\prime} .
$$

Also

$$
\left[X_{m \times n}\right] \cup\left\{\left[Y_{m \times n}\right] \Delta\left[Z_{m \times n}\right]\right\}=\max \left\{d_{h_{i}}, d_{h_{i}}^{\prime \prime}-d_{h_{i}}^{\prime}\right\} .
$$

If $\max \left\{d_{h_{i}}, d_{h_{i}}^{\prime \prime}-d_{h_{i}}^{\prime}\right\}=d_{h_{i}}$, then

$$
\left[X_{m \times n}\right] \cup\left\{\left[Y_{m \times n}\right] \Delta\left[Z_{m \times n}\right]\right\}=d_{h_{i}},
$$

which is the right hand side. Now we have to find the left hand side:

$$
\left[X_{m \times n}\right] \cup\left[Y_{m \times n}\right]=\max \left\{d_{h_{i}}, d_{h_{i}}^{\prime}\right\}=d_{h_{i}}^{\prime}=\left[Y_{m \times n}\right]
$$

Similarly

$$
\left[X_{m \times n}\right] \cup\left[Z_{m \times n}\right]=\left[Z_{m \times n}\right] .
$$

Thus

$$
\begin{gathered}
\left\{\left[X_{m \times n}\right] \cup\left[Y_{m \times n}\right]\right\} \Delta\left\{\left[X_{m \times n}\right] \cup\left[Z_{m \times n}\right]\right\}=\left[Y_{m \times n}\right] \Delta\left[Z_{m \times n}\right]=\max \left\{d_{h_{i}}^{\prime}, d_{h_{i}}^{\prime \prime}\right\}-\min \left\{d_{h_{i}}^{\prime}, d_{h_{i}}^{\prime \prime}\right\} . \\
\left\{\left[X_{m \times n}\right] \cup\left[Y_{m \times n}\right]\right\} \Delta\left\{\left[X_{m \times n}\right] \cup\left[Z_{m \times n}\right]\right\}=d_{h_{i}}^{\prime \prime}-d_{h_{i}}^{\prime} .
\end{gathered}
$$

Hence form (1) and (2), we have proved that

$$
\left[X_{m \times n}\right] \cup\left\{\left[Y_{m \times n}\right] \Delta\left[Z_{m \times n}\right]\right\} \neq\left\{\left[X_{m \times n}\right] \cup\left[Y_{m \times n}\right]\right\} \Delta\left\{\left[X_{m \times n}\right] \cup\left[Z_{m \times n}\right]\right\} .
$$

The proof of other cases is similar.

Theorem 4.10. For any three neutrosophic soft metric matrices $\left[X_{m \times n}\right],\left[Y_{m \times n}\right]$ and $\left[Z_{m \times n}\right]$, the intersection is not distributive over symmetric difference $\Delta$.

Proof. For any three neutrosophic soft metric matrices $\left[X_{m \times n}\right],\left[Y_{m \times n}\right]$ and $\left[Z_{m \times n}\right]$, we have to show that

$$
\left[X_{m \times n}\right] \cap\left\{\left[Y_{m \times n}\right] \Delta\left[Z_{m \times n}\right]\right\} \neq\left\{\left[X_{m \times n}\right] \cap\left[Y_{m \times n}\right]\right\} \Delta\left\{\left[X_{m \times n}\right] \cap\left[Z_{m \times n}\right]\right\} .
$$

To prove the above we assume that

$$
d_{h_{i}} \leq d_{h_{i}}^{\prime} \leq d_{h_{i}}^{\prime \prime}
$$


for all $d_{h_{i}} \in\left[X_{m \times n}\right], d_{h_{i}}^{\prime} \in\left[Y_{m \times n}\right]$ and $d_{h_{i}}^{\prime \prime} \in\left[Z_{m \times n}\right]$. Now

$$
\left[Y_{m \times n}\right] \Delta\left[Z_{m \times n}\right]=\max \left\{d_{h_{i}}^{\prime}, d_{h_{i}}^{\prime \prime}\right\}-\min \left\{d_{h_{i}}^{\prime}, d_{h_{i}}^{\prime \prime}\right\}=d_{h_{i}}^{\prime \prime}-d_{h_{i}}^{\prime}
$$

Also

$$
\left[X_{m \times n}\right] \cap\left\{\left[Y_{m \times n}\right] \Delta\left[Z_{m \times n}\right]\right\}=\min \left\{d_{h_{i}}, d_{h_{i}}^{\prime \prime}-d_{h_{i}}^{\prime}\right\},
$$

if $\min \left\{d_{h_{i}}, d_{h_{i}}^{\prime \prime}-d_{h_{i}}^{\prime}\right\}=d_{h_{i}} \neq 0$, then

$$
\left[X_{m \times n}\right] \cap\left\{\left[Y_{m \times n}\right] \Delta\left[Z_{m \times n}\right]\right\}=d_{h_{i}} .
$$

which is the left hand side. Now we have to find the right hand side:

$$
\left[X_{m \times n}\right] \cap\left[Y_{m \times n}\right]=\min \left\{d_{h_{i}}, d_{h_{i}}^{\prime}\right\}=d_{h_{i}}=\left[X_{m \times n}\right] .
$$

Similarly

$$
\left[X_{m \times n}\right] \cap\left[Z_{m \times n}\right]=\left[X_{m \times n}\right] .
$$

Thus

$$
\begin{gathered}
\left\{\left[X_{m \times n}\right] \cap\left[Y_{m \times n}\right]\right\} \Delta\left\{\left[X_{m \times n}\right] \cap\left[Z_{m \times n}\right]\right\}=\left[X_{m \times n}\right] \Delta\left[X_{m \times n}\right]=\max \left\{d_{h_{i}}, d_{h_{i}}\right\}-\min \left\{d_{h_{i}}, d_{h_{i}}\right\} \\
\left\{\left[X_{m \times n}\right] \cup\left[Y_{m \times n}\right]\right\} \Delta\left\{\left[X_{m \times n}\right] \cup\left[Z_{m \times n}\right]\right\}=d_{h_{i}}-d_{h_{i}}=0 .
\end{gathered}
$$

Hence from (3) and (4), we have proved that

$$
\left[X_{m \times n}\right] \cap\left\{\left[Y_{m \times n}\right] \Delta\left[Z_{m \times n}\right]\right\} \neq\left\{\left[X_{m \times n}\right] \cap\left[Y_{m \times n}\right]\right\} \Delta\left\{\left[X_{m \times n}\right] \cap\left[Z_{m \times n}\right]\right\} .
$$

The proof of other cases is similar.

Note. It is worth mentioning to add here that neutrosophic soft metric matrix is more general than a fuzzy soft matrix since the degree of truth membership function, intermediate membership function, and falsity membership function are added here yielding better choice in decision-making problems.

\section{Decision making algorithm}

In this section, we will discuss a real-life application of a neutrosophic soft metric matrix. Specifically, the neutrosophic soft metric matrix explains how to get a better and clear choice in decisionmaking problems.

We consider the problem of choosing the most appropriate house that a person will choose on the basis of his $n$ number of parameters out of $m$ number of houses. Let the $n$ number of choice parameters are $e_{1}, e_{2}, \ldots, e_{n}$ and the $m$ numbers of houses are $h_{1}, h_{2}, \ldots, h_{m}$. We also assume that the performance value of the houses corresponding to the parameters is represented by a neutrosophic soft set. The performance values may be arranged in the form of a matrix using the metric function $d_{h_{i}}$ called the "neutrosophic soft metric matrix".

We propose the following definitions for choice matrix and weighted choice matrix in view of a general decision-making problems and taking the idea of a neutrosophic soft metric matrix into account: 
Definition 5.1. If $\left[X_{m \times n^{\prime}}\right]=\left[d_{h_{i}}\right]_{m \times n^{\prime}}$ is a neutrosophic soft metric matrix, then the choice matrix of a neutrosophic soft metric matrix $\left[X_{m \times n^{\prime}}\right]$ is given by

$$
\left[C_{m \times n^{\prime}}\right]=\left(\frac{\sum_{i, j=1}^{l}\left(d_{h}\left(\epsilon_{\lambda}\left(e_{i}\right), \epsilon_{\lambda}\left(e_{j}\right)\right)\right)^{2}}{n(R)}\right)_{1 \times n},
$$

when weights are equal. $n(R)$ denotes the number of rows.

Definition 5.2. If $\left[X_{m \times n^{\prime}}\right]=\left[d_{h_{i}}\right]_{m \times n^{\prime}}$ is a neutrosophic soft metric matrix, then the weight matrix of a neutrosophic soft metric matrix $\left[X_{m \times n^{\prime}}\right]$ is given by

$$
\left[W_{m \times n^{\prime}}\right]=\left(\frac{\sum_{i, j=1}^{l} w_{i} w_{j}\left(d_{h}\left(\epsilon_{\lambda}\left(e_{i}\right), \epsilon_{\lambda}\left(e_{j}\right)\right)\right)^{2}}{\sum_{i=1}^{r} w_{i} \cdot \sum_{j=1}^{r} w_{j}}\right)_{1 \times n},
$$

where $w_{i}>0$ are weights.

Now, we have the following algorithm to give the better choice of house.

Algorithm

\section{Step 1.}

Consider the neutrosophic soft metric matrix for the houses $h_{1}, h_{2}, \ldots, h_{m}$ corresponding to the parameters $e_{1}, e_{2}, \ldots, e_{l}$, that is;

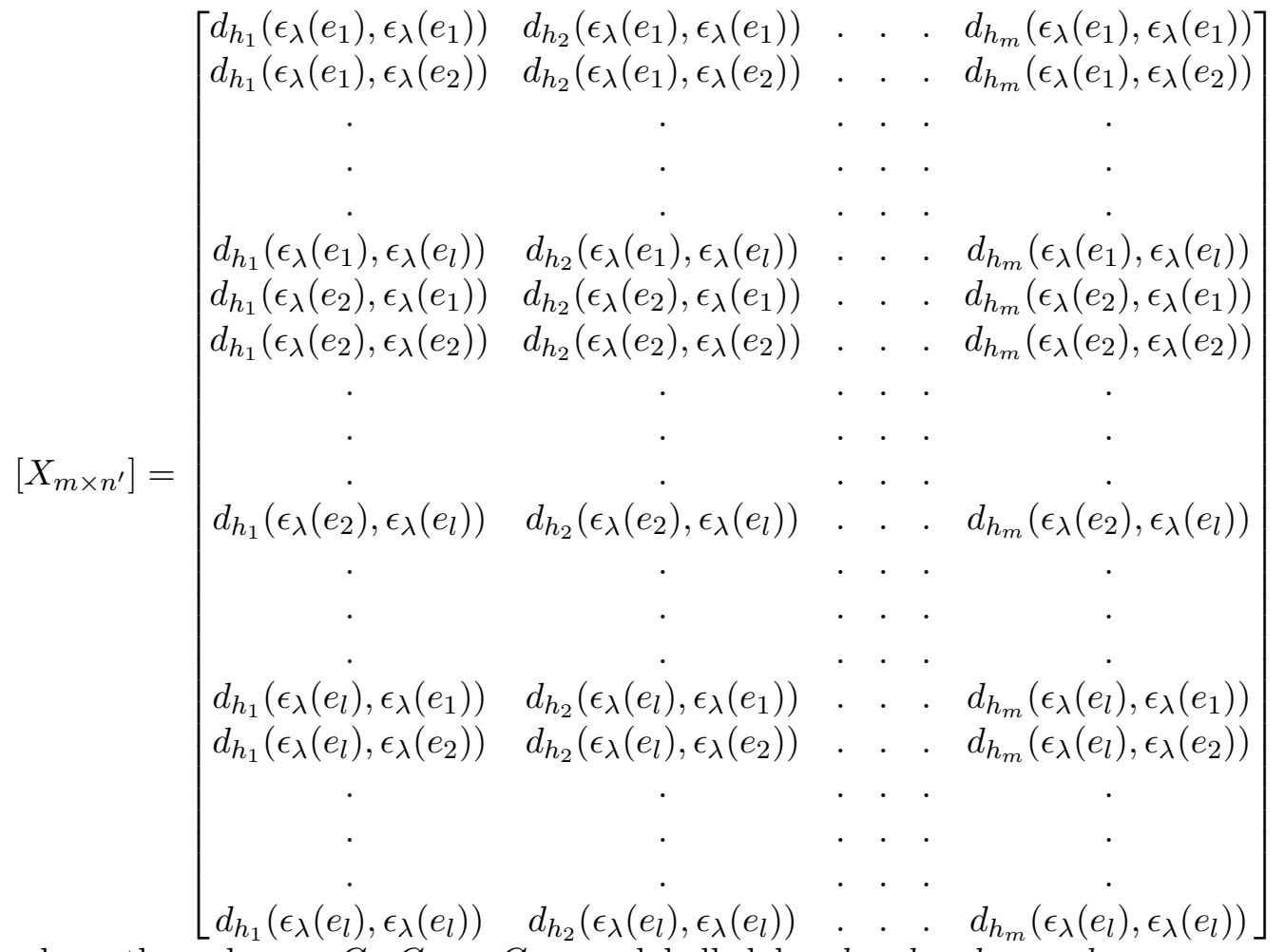

where the columns $C_{1}, C_{2}, \ldots, C_{m}$ are labelled by $d_{h_{1}}, d_{h_{2}}, d_{h_{3}}, \ldots, d_{h_{m}}$ corresponding to the houses $h_{1}, h_{2}, \ldots, h_{m}$, respectively.

\section{Step 2.}


Find the choice matrix of a neutrosphic soft metric matrix $\left[X_{m \times n}\right]$, that is,

$$
\left[C_{m \times n^{\prime}}\right]=\left(\frac{\sum_{i, j=1}^{l}\left(d_{h}\left(\epsilon_{\lambda}\left(e_{i}\right), \epsilon_{\lambda}\left(e_{j}\right)\right)\right)^{2}}{n(R)}\right)_{1 \times n} .
$$

\section{Step 3.}

Find the weighted choice matrix of a neutrosphic soft metric matrix $\left[X_{m \times n}\right]$, that is,

$$
\left[W_{m \times n^{\prime}}\right]=\left(\frac{\sum_{i, j=1}^{l} w_{i} w_{j}\left(d_{h}\left(\epsilon_{\lambda}\left(e_{i}\right), \epsilon_{\lambda}\left(e_{j}\right)\right)\right)^{2}}{\sum_{i=1}^{r} w_{i} \cdot \sum_{j=1}^{r} w_{j}}\right)_{1 \times n} .
$$

\section{Step 4.}

If the weights are equal, it means that we give an equal preference for all the parameters then the highest membership value from $\left[C_{m \times n^{\prime}}\right]$ shows the most suitable choice for the costomer.

If the weights are unequal, it means that the customer gives preference for the para- meter over the other parameters, then the highest membership value from $\left[W_{m \times n^{\prime}}\right]$ shows the most suitable choice for the customer.

\subsection{Application}

We consider the problem to select the most suitable house which Mr. Aslam is going to choose on the basis of the parameters $e_{1}=$ cheap, $e_{2}=$ beautiful, $e_{3}=$ in good repairing. Let us assume that the set of three houses are $U=\left\{h_{1}, h_{2}, h_{3}\right\}$. We also assume that the performance values of the houses corresponding to the parameters are:

$$
\begin{gathered}
\epsilon_{\lambda}(\text { cheap })=\epsilon_{\lambda}\left(e_{1}\right)=\left\{\left\langle h_{1}, 0.5,0.6,0.9\right\rangle,\left\langle h_{2}, 0.2,0.5,0.3\right\rangle,\left\langle h_{3}, 0.8,0.4,1\right\rangle\right\}, \\
\epsilon_{\lambda} \text { (beautiful) }=\epsilon_{\lambda}\left(e_{2}\right)=\left\{\left\langle h_{1}, 0.8,0.2,0.6\right\rangle,\left\langle h_{2}, 0.8,0.5,0.7\right\rangle,\left\langle h_{3}, 0.4,0.9,0.1\right\rangle\right\}, \\
\epsilon_{\lambda}(\text { in good repairing })=\epsilon_{\lambda}\left(e_{3}\right)=\left\{\left\langle h_{1}, 0.2,0.5,0.8\right\rangle,\left\langle h_{2}, 1,0.9,0.1\right\rangle,\left\langle h_{3}, 0.4,0.6,0.8\right\rangle\right\} .
\end{gathered}
$$

Now, on the basis of algorithm defined above we have the following steps:

\section{Step 1.}

Find the neutrosophic soft metric matrix for the houses $h_{1}, h_{2}, h_{3}$ corresponding to the parameters $e_{1}, e_{2}, e_{3}$, that is; for house $h_{1}$ we have

$$
\begin{aligned}
& d_{h_{1}}\left(\epsilon_{\lambda}\left(e_{1}\right), \epsilon_{\lambda}\left(e_{1}\right)\right)=0, d_{h_{1}}\left(\epsilon_{\lambda}\left(e_{1}\right), \epsilon_{\lambda}\left(e_{2}\right)\right)=0.3, d_{h_{1}}\left(\epsilon_{\lambda}\left(e_{1}\right), \epsilon_{\lambda}\left(e_{3}\right)\right)=0.2, \\
& d_{h_{1}}\left(\epsilon_{\lambda}\left(e_{2}\right), \epsilon_{\lambda}\left(e_{1}\right)\right)=0.3, d_{h_{1}}\left(\epsilon_{\lambda}\left(e_{2}\right), \epsilon_{\lambda}\left(e_{2}\right)\right)=0, d_{h_{1}}\left(\epsilon_{\lambda}\left(e_{2}\right), \epsilon_{\lambda}\left(e_{3}\right)\right)=0.4, \\
& d_{h_{1}}\left(\epsilon_{\lambda}\left(e_{3}\right), \epsilon_{\lambda}\left(e_{1}\right)\right)=0.2, d_{h_{1}}\left(\epsilon_{\lambda}\left(e_{3}\right), \epsilon_{\lambda}\left(e_{2}\right)\right)=0.4, d_{h_{1}}\left(\epsilon_{\lambda}\left(e_{3}\right), \epsilon_{\lambda}\left(e_{3}\right)\right)=0 . \\
& \text { For house } h_{2} \text { we have } \\
& d_{h_{2}}\left(\epsilon_{\lambda}\left(e_{1}\right), \epsilon_{\lambda}\left(e_{1}\right)\right)=0, d_{h_{2}}\left(\epsilon_{\lambda}\left(e_{1}\right), \epsilon_{\lambda}\left(e_{2}\right)\right)=0.3, d_{h_{2}}\left(\epsilon_{\lambda}\left(e_{1}\right), \epsilon_{\lambda}\left(e_{3}\right)\right)=0.5, \\
& d_{h_{2}}\left(\epsilon_{\lambda}\left(e_{2}\right), \epsilon_{\lambda}\left(e_{1}\right)\right)=0.3, d_{h_{2}}\left(\epsilon_{\lambda}\left(e_{2}\right), \epsilon_{\lambda}\left(e_{2}\right)\right)=0, d_{h_{2}}\left(\epsilon_{\lambda}\left(e_{2}\right), \epsilon_{\lambda}\left(e_{3}\right)\right)=0.4, \\
& d_{h_{2}}\left(\epsilon_{\lambda}\left(e_{3}\right), \epsilon_{\lambda}\left(e_{1}\right)\right)=0.5, d_{h_{2}}\left(\epsilon_{\lambda}\left(e_{3}\right), \epsilon_{\lambda}\left(e_{2}\right)\right)=0.4, d_{h_{2}}\left(\epsilon_{\lambda}\left(e_{3}\right), \epsilon_{\lambda}\left(e_{3}\right)\right)=0 . \\
& \text { For house } h_{3} \text { we have } \\
& d_{h_{3}}\left(\epsilon_{\lambda}\left(e_{1}\right), \epsilon_{\lambda}\left(e_{1}\right)\right)=0, d_{h_{3}}\left(\epsilon_{\lambda}\left(e_{1}\right), \epsilon_{\lambda}\left(e_{2}\right)\right)=0.6, d_{h_{3}}\left(\epsilon_{\lambda}\left(e_{1}\right), \epsilon_{\lambda}\left(e_{3}\right)\right)=0.3, \\
& d_{h_{3}}\left(\epsilon_{\lambda}\left(e_{2}\right), \epsilon_{\lambda}\left(e_{1}\right)\right)=0.6, d_{h_{3}}\left(\epsilon_{\lambda}\left(e_{2}\right), \epsilon_{\lambda}\left(e_{2}\right)\right)=0, d_{h_{3}}\left(\epsilon_{\lambda}\left(e_{2}\right), \epsilon_{\lambda}\left(e_{3}\right)\right)=0.3, \\
& d_{h_{3}}\left(\epsilon_{\lambda}\left(e_{3}\right), \epsilon_{\lambda}\left(e_{1}\right)\right)=0.3, d_{h_{3}}\left(\epsilon_{\lambda}\left(e_{3}\right), \epsilon_{\lambda}\left(e_{2}\right)\right)=0.3, d_{h_{3}}\left(\epsilon_{\lambda}\left(e_{3}\right), \epsilon_{\lambda}\left(e_{3}\right)\right)=0 .
\end{aligned}
$$


The neutrosophic soft metric matrix is

$$
\left[X_{3 \times 9}\right]=\left[\begin{array}{ccc}
0 & 0 & 0 \\
0.3 & 0.3 & 0.6 \\
0.2 & 0.5 & 0.3 \\
0.3 & 0.3 & 0.6 \\
0 & 0 & 0 \\
0.4 & 0.4 & 0.3 \\
0.2 & 0.5 & 0.3 \\
0.4 & 0.4 & 0.3 \\
0 & 0 & 0
\end{array}\right] .
$$

\section{Step 2.}

Find the choice matrix of a neutrosphic soft metric matrix $\left[X_{m \times n}\right]$, that is,

$$
\left[C_{1 \times 3}\right]=\left[\begin{array}{lll}
0.06 & 0.11 & 0.12
\end{array}\right] .
$$

\section{Step 3.}

If the weights $0.2,0.6,0.2$ are given for the parameters cheap, beautiful, in good repairing, respectively, then the weighted choice matrix for $\left[X_{9 \times 3}\right]$ is as:

$$
\left[W_{1 \times 3}\right]=\left[\begin{array}{lll}
0.04 & 0.08 & 0.1152
\end{array}\right] \text {. }
$$

\section{Step 4.}

If the weights are equal, then the choice matrix $\left[C_{1 \times 3}\right]$ obtained in Step 2, we have the highest membership value 0.12 from $\left[C_{1 \times 3}\right]$ shows that the most suitable choice for the customer is $h_{3}$. If the weights are unequal, it means that the customer gives preference for the parameter "beautiful" over the other parameters, then the highest membership value is 0.1152 from $\left[W_{1 \times 3}\right]$ shows that the most suitable choice for the costumer is again $h_{3}$.

\section{Comparison}

The soft set, fuzzy soft set, complex fuzzy soft set, fuzzy soft matrix, complex fuzzy soft matrix, neutrosophic soft set, etc has many applications, particularly in decision-making problems. Here we presented the application of neutrosophic soft metric matrix. In this practical application, how to choose a suitable model is the main issue. We studied this approach in depth and introduced an algorithm using the matrix called the neutrosophic soft metric matrix. It is important to note here that the neutrosophic soft metric matrix is more general than the fuzzy soft matrix since the degree of true membership function, the intermediate membership function, and the falsity membership function are added here, giving better choice in decision-making problems. The model presented in this paper for identifying a suitable choice is a more effective method in decision-making problems. Through this model, we compared clearly the max value of each choice with the max value of a suitable choice. However our designed model is not a perfect one, it stuck with a deficiency of theoretical support. The concept of a neutrosophic soft metric matrix for a neutrosophic soft set may be useful for applications. Therefore, it will be significant for future work.

\section{Conclusion}

In this paper, we introduced the concept of a neutrosophic soft metric matrix and defined different types of matrices in neutrosophic soft set theory along with examples. We have presented some 
basic results and examples of these matrices. Moreover, we have discussed the application of neutrosophic soft metric matrices in decision-making problems. Moreover, this work and further study of neutrosophic soft metric matrices will give a new direction of applications in different fields of science and engineering.

\section{Acknowledgments}

This work is financially supported by the Higher Education Commission of Pakistan (Grant No: 7750/Federal/NRPU/R\&D/HEC/2017).

Conflict of Interests

The authors declare that they have no conflict of interests.

Authorship Contributions: All authors contributed equally.

\section{References}

[1] H. Aktas, N. Cagman, Soft sets and soft groups, Information Sciences, 177(3) (2007), 27262735

[2] M.I. Ali, F. Feng, X. Liu, W.K. Min, M. Shabir, On some new operations in soft set theory, Computers and Mathematics with Applications, 57(9) (2009), 1547-1553.

[3] M. Ali, L.H. Son, I. Deli, N.D. Tien, Bipolar neutrosophic soft sets and applications in decision making, Journal of Intelligent and Fuzzy Systems, 33(6) (2017), 4077-4087.

[4] K.T. Atanassov, Intuitionistic fuzzy sets, In Intuitionistic Fuzzy Sets, Springer, (1999), 1-137.

[5] A. Aygunoglu, H. Aygun, Introduction to fuzzy soft groups, Computers and Mathematics with Applications, 58(6) (2009), 1279-1286.

[6] S. Broumi, Generalized neutrosophic soft set, International Journal of Computer Science, Engineering and Information Technology, 3(2) (2013), 17-30.

[7] S. Broumi, F. Smarandache, Intuitionistic neutrosophic soft set, Journal of Information and Computing Science, 8(2) (2013), 130-140.

[8] N. Çağman, S. Enginoğlu, Soft matrix theory and its decision making, Computers and Mathematics with Applications, 59 (2010), 3308-3314.

[9] N. Çağman, S. Enginoğlu, Fuzzy soft matrix theory and its applications in decision making, Iranian Journal of Fuzzy Systems, 9(1) (2012), 109-119.

[10] I. Deli, Interval-valued neutrosophic soft sets and its decision making, International Journal of Machine Learning and Cybernetics, 8(2) (2017), 665-676.

[11] I. Deli, S. Broumi, Neutrosophic soft matrices and NSM-decision making, Journal of Intelligent and Fuzzy Systems, 28(5) (2015), 2233-2241.

[12] F. Feng, Y.B. Jun, X.Y. Liu, L.F.Li, An adjustable approach to fuzzy soft set based decision making, Journal of Computational and Applied Mathematics, 234 (2010), 10-20. 
[13] F. Feng, Y.B. Jun, X.Z. Zhao, Soft semirings, Computers and Mathematics with Applications, 56 (2008), 2621-2628.

[14] F. Feng, C. Li, B. Davvaz, M.I. Ali, Soft sets combined with fuzzy sets and rough sets: A tentative approach, Soft Computing, 14 (2010), 899-911.

[15] F. Feng, X.Y. Liu, V. Leoreanu-Fotea, Y.B. Jun, Soft sets and rough sets, Information Sciences, 181 (2011), 1125-1137.

[16] S. Jha, R. Kumar, J.M. Chatterjee, M. Khari, N. Yadav, F. Smarandache, Neutrosophic soft set decision making for stock trending analysis, Evolving Systems, 10(4) (2019), 621-627.

[17] F. Karaaslan, Neutrosophic soft sets with applications in decision making, Infinite Study, 2014.

[18] M. Khan, A.N. Saima, S.Z. Song, J.U. Youngbae, Complex fuzzy soft matrices with applications, Hacettepe Journal of Mathematics and Statistics, 149(2) (2020), 676-83.

[19] J. Maiers, Y.S. Sherif, Applications of fuzzy set theory, IEEE Transactions on Systems, Man, and Cybernetics, 1 (1985), 175-189.

[20] P.K. Maji, Neutrosophic soft set, Annals of Fuzzy Mathematics and Informatics, 5(1) (2013), $157-168$.

[21] P.K. Maji, R.K. Biswas, A. Roy, Fuzzy soft sets, Journal of Fuzzy Mathematics, (2001), 589-602.

[22] P.K. Maji, R.K. Biswas, A. Roy, Intuitionistic fuzzy soft sets, Journal of Fuzzy Mathematics, $9(3)$ (2001), 677-692.

[23] P.K. Maji, R.K. Biswas, A. Roy, An application of soft sets in a decision making problem, Computers and Mathematics with Applications, 44 (2002), 1077-1083.

[24] P.K. Maji, R.K. Biswas, A. Roy, Soft set theory, Computers and Mathematics with Applications, 45 (2003), 555-562.

[25] S. Manna, T.M. Basu, S.K. Mondal, A soft set based VIKOR approach for some decisionmaking problems under complex neutrosophic environment, Engineering Applications of Artificial Intelligence, 89 (2020), 103432.

[26] D. Molodtsov, Soft set theory-first results, Computers and Mathematics with Applications, 37 (1999), 19-31.

[27] M.A. Qamar, N. Hassan, An approach toward a Q-neutrosophic soft set and its application in decision making, Symmetry, 11(2) (2019), 139.

[28] D. Ramot, R. Milo, M. Friedman, A. Kandel, Complex fuzzy sets, IEEE Transactions on Fuzzy Systems, 10(2) (2002), 171-186.

[29] M. Saqlain, M.N. Jafar, M. Riaz, A new approach of neutrosophic soft set with generalized fuzzy TOPSIS in application of smart phone selection, Neutrosophic Sets and Systems, 32 (2020), 307-316.

[30] F. Smarandache, Neutrosophy: Neutrosophic probability, set, and logic: Analytic synthesis and synthetic analysis, American Research Press, 1998. 
[31] F. Smarandache, Neutrosophic set-a generalization of the intuitionistic fuzzy set, International Journal of Pure and Applied Mathematics, 24(3) (2005), 287-297.

[32] L. A. Zadeh, Fuzzy sets, Information and Control, 8 (1965), 338-353. 